\title{
REVIEW OF THE COLLECTED WORKS OF JOHN TATE
}

\author{
J. S. MILNE
}

Abstract. This is a review of Collected Works of John Tate. Parts I, II, edited by Barry Mazur and Jean-Pierre Serre. American Mathematical Society, Providence, Rhode Island, 2016.

For several decades it has been clear to the friends and colleagues of John Tate that a "Collected Works" was merited. The award of the Abel Prize to Tate in 2010 added impetus, and finally, in Tate's ninety-second year we have these two magnificent volumes, edited by Barry Mazur and Jean-Pierre Serre. Beyond Tate's published articles, they include five unpublished articles and a selection of his letters, most accompanied by Tate's comments, and a collection of photographs of Tate. For an overview of Tate's work, the editors refer the reader to [4. Before discussing the volumes, I describe some of Tate's work.

\section{HeCke $L$-SERIES AND TATE'S THesis}

Like many budding number theorists, Tate's favorite theorem when young was Gauss's law of quadratic reciprocity. When he arrived at Princeton as a graduate student in 1946, he was fortunate to find there the person, Emil Artin, who had discovered the most general reciprocity law, so solving Hilbert's ninth problem. By 1920, the German school of algebraic number theorists (Hilbert, Weber, ...) together with its brilliant student Takagi had succeeded in classifying the abelian extensions of a number field $K$ : to each group $I$ of ideal classes in $K$, there is attached an extension $L$ of $K$ (the class field of $I$ ); the group $I$ determines the arithmetic of the extension $L / K$, and the Galois group of $L / K$ is isomorphic to I. Artin's contribution was to prove (in 1927) that there is a natural isomorphism from $I$ to the Galois group of $L / K$. When the base field contains an appropriate root of 1 , Artin's isomorphism gives a reciprocity law, and all possible reciprocity laws arise this way.

In the 1930s, Chevalley reworked abelian class field theory. In particular, he replaced "ideals" with his "idèles" which greatly clarified the relation between the local and global aspects of the theory. For his thesis, Artin suggested that Tate do the same for Hecke $L$-series. When Hecke proved that the abelian $L$-functions of number fields (generalizations of Dirichlet's $L$-functions) have an analytic continuation throughout the plane with a functional equation of the expected type, he saw that his methods applied even to a new kind of $L$-function, now named after him. Once Tate had developed his harmonic analysis of local fields and of the idèle group, he was able prove analytic continuation and functional equations for all the relevant $L$-series without Hecke's complicated theta-formulas.

Received by the editors September 5, 2016.

2010 Mathematics Subject Classification. Primary 01A75, 11-06, 14-06. 
As Kudla writes:

Tate provides an elegant and unified treatment of the analytic continuation and functional equation of Hecke $L$-functions. The power of the methods of abelian harmonic analysis in the setting of Chevalley's adèles/idèles provided a remarkable advance over the classical techniques used by Hecke. ... In hindsight, Tate's work may be viewed as giving the theory of automorphic representations and $L$-functions of the simplest connected reductive group $G=\mathrm{GL}(1)$, and so it remains a fundamental reference and starting point for anyone interested in the modern theory of automorphic representations.

Tate's thesis completed the re-expression of the classical class field theory in terms of idèles. In this way, it marked the end of one era and the start of a new.

\section{Galois cohomology and the Tate-Nakayama isomorphism}

Tate was awarded the Cole Prize by the AMS in 1956 for his paper determining the higher dimensional cohomology groups of class field theory. For this, Tate observed that it is possible to regard the homology groups of a finite group as cohomology groups with negative coefficients and so obtain a sequence of cohomology groups indexed by $\mathbb{Z}$. He then constructed a canonical sequence of isomorphisms

$$
H^{i}(G, \mathbb{Z}) \rightarrow H^{i+2}(G, C), \quad i \in \mathbb{Z},
$$

whenever $G$ is a finite group and $C$ is a $G$-module satisfying certain hypotheses. If $G$ is the Galois group of a finite extension $L / K$ of number fields, then the idèle class group $C$ of $L$ satisfies the hypotheses, and so Tate's theorem describes the groups $H^{i}(G, C)$ in terms of the known groups $H^{i}(G, \mathbb{Z})$. Tate's isomorphism becomes Artin's reciprocity isomorphism when $G$ is abelian and $i=-1$.

Tate's isomorphism was generalized by Nakayama, and then again by Tate [ 8 , and it is now called the Tate-Nakayama isomorphism. It has become a basic tool in algebraic number theory.

Tate continued his study of the applications of the cohomology of finite groups to arithmetic, and he introduced the cohomology of profinite groups (which he initially called groups of Galois type). He proved duality theorems and results on Euler characteristics, and he introduced and studied the notion of the cohomological dimension of fields. He announced some of his results in his 1962 ICM talk, but the material grew faster than his ability to write it up. Fortunately, others (Serre, Lang, ...) accomplished this task for him. Tate's article [11] is a brief introduction to this material.

\section{LOCAL CLASS FIELD THEORY AND LUBIN-TATE SPACES}

By the mid 1950s, abelian class field theory could be considered a mature field. However, some problems remained. One was that of explicitly generating all the fields shown to exist by the theory. For number fields, this is Hilbert's twelfth problem, for which there is still only a partial solution. For local fields, the problem was spectacularly solved by Lubin and Tate. 
Tate's student Lubin had completed his thesis on one-parameter formal Lie groups in 1963. In early 1964, Tate wrote:

[From Lubin's results] we get a homomorphism $G_{K} \rightarrow U_{K}=$ units in $K$. Restricting this to the inertia group $G_{K_{u}}$, we get a homomorphism $G_{K_{u}} \rightarrow U_{K}$, which is probably canonical by the unicity remarks above, and which it is impossible to doubt is in fact the reciprocity law isomorphism (or its negative!) ... The miracle seems to be that once one abandons algebraic groups, and goes to formal groups, the theory of complex multiplication applies universally (locally). (Letter to Serre, January 10, 1964; CW, L8.)

Local class field theory provides, for each prime element $\pi$ of a local field $K$, a totally ramified abelian extension $K_{\pi}$ of $K$ such that every abelian extension of $K$ is contained in the composite of $K_{\pi}$ with an unramified extension. For each $\pi$, Lubin and Tate give a remarkably simple construction of a tower of extensions of $K$ whose union is $K_{\pi}$.

Lubin and Tate went on to study the moduli spaces of one-parameter formal Lie groups. These turned out to be more important than the authors imagined at the time. The towers obtained by adding level structures were studied by Drinfeld. The "Lubin-Tate spaces" obtained in this way form a local analogue of Shimura varieties, and played a role, for example, in the proof by Harris and Taylor of nonabelian local class field theory for $\mathrm{GL}_{n}$ (the local Langlands conjecture for $\mathrm{GL}_{n}$ ).

\section{Abelian varieties and the Artin-Tate conjecture}

For a complex analyst, an abelian variety is a complex torus $A=\mathbb{C}^{g} /\{$ lattice $\}$ admitting an embedding into projective space by theta functions. In giving substance to his proofs of the function field analogues of the Riemann and Artin conjectures (announced in 1940), Weil developed the theory of abelian varieties over arbitrary fields (1948). In the subsequent years, much of the theory of elliptic curves was extended to abelian varieties of arbitrary dimension. For example, every embedding of an abelian variety into projective space defines a theory of heights on the abelian variety, but an abelian variety has many such embeddings. Néron conjectured that there is a canonical height satisfying a certain quadraticity condition, and Tate found a remarkably simple proof of Néron's conjecture.

In an important series of papers (1959-65), Cassels reworked the arithmetic theory of elliptic curves over number fields. In particular, he proved some duality theorems, including one showing that the mysterious Tate-Shafarevich group of the curve has order a square if finite. Tate extended the results of Cassels to abelian varieties over number fields.

As a result of some of the first computer experiments, Birch and SwinnertonDyer were led to conjecture that the rank of the group of $\mathbb{Q}$-points of an elliptic curve over $\mathbb{Q}$ is equal to the order of the zero of its $L$-function at $s=1$ (BSD1). Later they found a conjectural formula for the first Taylor coefficient of the $L$ function in terms other arithmetic invariants of the curve, including the order of the Tate-Shafarevich group and the discriminant of the height pairing (BSD2). Tate recognized that the natural setting for the conjectures is an abelian variety over a global field, and his proof of Néron's conjecture allowed him to state them in this generality. 
For a surface $X$ fibered in elliptic curves over a curve over a finite field, Tate recognized that the function field version of (BSD1) for the generic fiber implied that the rank of the Picard group of $X$ equals the order of the pole of its zeta function at $s=1$. This is one of the observations that led him to the Tate conjecture (see the next section). With Michael Artin, he examined the significance of (BSD2) for the surface. This led them to state the Artin-Tate conjecture which relates the behavior near $s=1$ of the zeta function of a surface over a finite field to other arithmetic invariants of the surface, including the order of its Brauer group.

\section{The Tate COnjecture}

The Tate conjecture occupies the same central place in arithmetic algebraic geometry that the Hodge conjecture occupies in complex algebraic geometry. For an algebraic variety over $\mathbb{C}$, the Hodge conjecture characterizes the $\mathbb{Q}$-subspace of the usual cohomology groups conjecturally spanned by the classes of algebraic cycles. For an algebraic variety over a general algebraically closed field $k$, the Tate conjecture characterizes the $\mathbb{Q}_{\ell}$-subspace of the $\ell$-adic étale cohomology conjecturally spanned by the algebraic classes.

Consider an algebraic variety $X$ (smooth and projective) over an algebraically closed field $k$. An algebraic cycle is defined by finitely many polynomials having only finitely many coefficients, and so it and its cohomology class are defined over a subfield of $k$ finitely generated over the prime field. The Tate conjecture says that this condition can be used to characterize the $\mathbb{Q}_{\ell}$-space spanned by the algebraic classes. There are many variants and special cases of the conjecture. One is the relation between the Picard number of a surface over a finite field and its zeta function noted above. Another (the isogeny conjecture) is that for abelian varieties $A$ and $B$ over a field $k$ finitely generated over the prime field, the map

$$
\operatorname{Hom}(A, B) \otimes \mathbb{Z}_{\ell} \rightarrow \operatorname{Hom}\left(T_{\ell} A, T_{\ell} B\right)^{G_{k}}
$$

is an isomorphism; here $T_{\ell} A$ is the first $\ell$-adic homology group of $A$ (its Tate group) and $G_{k}$ is the absolute Galois group of $k$. A third, particularly favored by Tate, is the following statement:

If $X$ is a regular scheme of finite type over $\mathbb{Z}$, then the order of $\zeta(X, s)$ at the point $s=\operatorname{dim} X-1$ is equal to $\operatorname{rank} H^{0}\left(X, \mathcal{O}_{X}^{*}\right)-$ $\operatorname{rank} H^{1}\left(X, \mathcal{O}_{X}^{*}\right)$.

Tate accumulated evidence for his conjectures during 1963, spoke about them at conferences in late 1963 and mid-1964, and published them in the proceedings of the first conference [7]. When asked about the origin of the Tate conjecture, Tate responded:

Early on I somehow had the idea that the special case about endomorphisms of abelian varieties over finite fields might be true. A bit later I realized that a generalization fit perfectly with the function field version of the Birch and Swinnerton-Dyer conjecture. Also it was true in various particular examples which I looked at and gave a heuristic reason for the Sato-Tate distribution. So it seemed a reasonable conjecture.

In perhaps his most beautiful paper, Tate proved the isogeny conjecture over finite fields in 1966, and Artin and Swinnerton-Dyer proved Tate's conjecture for elliptic $K 3$ surfaces over finite fields in 1973. Since then there has been little 
progress - it took another forty years and the efforts of several mathematicians to complete the proof of the Tate conjecture for $K 3$ surfaces over finite fields. The Hodge conjecture is known in degree 2, and there is strong evidence for the Tate conjecture in the same degree, but beyond that both statements are generally considered to be open questions. However, the conjectures guide later research. For example, the form of the conjecture of Langlands and Rapoport, which is basic to an understanding of the zeta function of a general Shimura variety, is shaped by the two conjectures.

\section{Rigid AnAlytic SPACES AND the TAte CURVE}

The topology on the field $K=\mathbb{Q}_{p}$ of $p$-adic numbers is very different from the real topology. For example, every disk in $K$ can be written as a disjoint union of arbitrarily many open-closed smaller disks, and so any local definition of "analytic function" leads to too many functions. Moreover, it is not possible to represent an elliptic curve as a quotient of $K$ by a discrete lattice because $K$ has no discrete subgroups.

However, there is an alternative representation of an elliptic curve $E$ over $\mathbb{C}$, namely, $E(\mathbb{C})=\mathbb{C}^{\times} / q^{\mathbb{Z}}$ for some $q \in \mathbb{C}$. When Tate took $q$ to be an element of a $p$-adic field $K$ with $|q|<1$, he was "amazed and thrilled" to find that, suitably normalized, the classical power series converge and give an elliptic curve $E$ over $K$ whose points satisfy $E(K)=K^{\times} / q^{\mathbb{Z}}$. Curves arising in this way are called Tate curves. Higher dimensional analogues of Tate's construction have been found, and are essential to an understanding of the compactification of the moduli schemes of abelian varieties.

Although this only realized $E(K)$ as a quotient of abstract groups, Tate understood from the start that the quotient should live in some category of "analytic spaces":

Finally, and most important, this last theorem and probably many other things that are hard to prove at present, would become obvious if one really had a theory of analytic and meromorphic functions in complete non-archimedean fields ... How does one get around the total disconnectedness to get some kind of global theory? One really must try to make sense out of Krasner's stuff. I have not yet had the courage, however. But everything points to the existence of $\mathfrak{p}$-adic analytic continuation (letter to Serre, August 4, 1959).

When consulted by Tate, Grothendieck was initially negative:

Nor do I have the impression of having understood this theorem at all; it does nothing more than exhibit, via brute formulas, a certain isomorphism of analytic groups (letter to Serre, October 19, 1961).

Only when Tate began constructing an underlying theory of analytic spaces did Grothendieck become more positive and helpful.

So how did Tate resolve the problem noted above? He first defined a local theory in which the objects are ringed spaces $\left(X, \mathcal{O}_{X}\right)$ whose underlying set $X$ has a $p$-adic topology. He then defined a collection of "admissible" open subsets and "admissible" open coverings. Although the admissible open sets and coverings do not form a topology in the usual sense, they satisfy the conditions necessary to support a sheaf theory (they form a Grothendieck topology). In this way, Tate 
was able define global objects and a "locally" defined notion of analytic function: a function is analytic if it becomes analytic on the open subsets of some admissible covering. An acyclicity theorem of Tate shows that this gives a good theory.

Tate reported on his work in a series of letters to Serre, who had them typed and distributed by I.H.E.S. They soon attracted the attention of the German school of complex analytic geometers, and already by 1984 a comprehensive account of the theory required a book of over 400 pages. Tate's notes were eventually published as 9]. There have been a number of extensions of Tate's theory (Raynaud, Berkovich, ...), and $p$-adic analytic spaces are now as much a part of the landscape of arithmetic geometry as real analytic spaces.

\section{MAny other Aspects of TATE'S WORK}

The above describes a part of Tate's work during the 1950s and 1960s. Tate also worked on many different aspects of elliptic curves throughout his career, the $K$ theory of number fields, the Stark conjectures, commutative and noncommutative algebra, and other topics.

I now discuss the Collected Works (CW).

\section{CW: ThE UNPUBlished PAPERS}

Tate's unpubished papers include the following. Paper 13 is a three-page proof of the Riemann hypothesis for function fields (Weil's theorem) using ideas of Mattuck, Tate, and Grothendieck. Paper 22 presents the notes of a seminar held as part of the 1964 Woods Hole conference in which Lubin, Serre, and Tate discuss some of their results on formal groups and abelian varieties, including Tate's proof of the Serre-Tate lifting theorem (see also letter L8 and comments). Paper 38 is a note in which Tate computes a certain subgroup (the tame kernel) of the $K_{2}$ of a number field. Paper 51 is a seven-page proof of the $\mathfrak{p}$-adic case of the Harish-Chandra transform for $\mathrm{GL}_{r}$. Paper 53 is a manuscript in which Tate states and partially proves a conjecture extending the theorems of Shimura and Taniyama on abelian varieties with complex multiplication to all automorphisms over $\mathbb{Q}$.

\section{CW: The LetTers}

Two of these letters are to Dwork. In the first, Tate describes a parameterization of the $p$-adic points of an elliptic curve, and he states a relation between a constant occurring in the parameterization and the zeta function of the curve. This was the spark that led to Dwork's remarkable $p$-adic proof of the rationality of the zeta function of a variety over a finite field. This is well explained in Tate's comments on the letter and in [2].

A letter to Springer explains Tate's proof, using Ext's, of the arithmetic duality theorems for finite modules announced in his 1962 ICM talk 6]. Another to Birch discusses what is now called the Birch-Tate conjecture, and a letter to Atkin describes the construction by Tate and his students of the first example of an Artin $L$-series known to be holomorphic even though no power of it is a product of an abelian $L$-series.

The remaining letters are a selection from the remarkable trove [1]. To comment on these would require an essay in itself. 


\section{CW: TATE'S COMMENTS ON THE PAPERS}

Some of Tate's comments are corrections. All but three of these can be considered misprints. Of the three, one is a muddled proof of a (correct) lemma (CW I, p. 116), one is a slightly misstated corollary (CW I, p. 424), and only one can be considered serious. In his 1962 ICM talk [6], Tate overstated what his methods give concerning the strict cohomological dimension of the various Galois groups attached to number fields (CW I, p. 180).

Some discuss the origins of papers, for example ([10]),

This is my first of several papers on $K_{2}$ of fields. I was introduced to the subject by Hyman Bass in the Fall of 1968, when we were both in Paris. He explained $K_{2}$ of fields to me via Steinberg symbols and the results of Calvin Moore described in $\S 2$ of this paper, then asked if I could see what $K_{2}(\mathbb{Q})$ was. I was able to answer his question...

While most of us are inclined to inflate our contributions, Tate's inclination seems to be the reverse. For example, he writes concerning [5]:

This paper was written by Serre. He probably put my name on it because we had discussed some of the contents. But the discussion was very one-sided. He did most of the talking and I the listening.

Tate's letter to Serre, June 14, 1967, suggests a somewhat greater engagement. Tate had received "état 0" of the paper written by Serre and writes "I am now about ready to tackle the état 1 of our paper". What follows is a series of comments and criticisms ("concerning your idiotic Lemma 1"), including the suggestion to replace "almost good reduction" with "potentially good reduction" (accepted).

Readers may be surprised to learn that Tate's contribution to a 2006 paper with Barry Mazur and William Stein [3] was "in doing the computer experiments discussed in section 6". However, Tate's letters to Serre (CW I, L18, L19, L20) reveal that as early as 1979, he was programming on a "hand-held Hewlett-Packard calculator on which one could write and run programs of 50 or fewer steps."

Concerning his great paper [7, Tate writes:

This paper contains many optimistic thoughts, which have become known as the "Tate conjectures".

This is far from the exuberant way in which Tate announced the conjectures in a letter to Serre more than five decades earlier:

At any rate, I am by now utterly convinced of the truth of THE Conjecture.

\section{About the Author}

J. S. Milne is professor emeritus of mathematics at the University of Michigan.

\section{REFERENCES}

[1] Correspondance Serre-Tate. Vol. I, II. Edited, and with notes and commentaries by Pierre Colmez and Jean-Pierre Serre. Documents Mathématiques (Paris), 13, 14. Société Mathématique de France, Paris, 2015.

[2] N. M. Katz and J. Tate, Bernard Dwork (1923-1998), Notices Amer. Math. Soc. 46 (1999), no. 3, 338-343; CW 73. MR.1669973 
[3] B. Mazur, W. Stein, and J. Tate, Computation of p-adic heights and log convergence, Doc. Math. Extra Vol. (2006), 577-614; CW 78. MR2290599

[4] J. S. Milne, "The Work of John Tate", The Abel Prize 2008-2012 (Helge Holden and Ragni Piene, editors), pp. 259-340, Springer, Heidelberg, 2014. MR3185030

[5] J.-P. Serre and J. Tate, Good reduction of abelian varieties, Ann. of Math. (2) 88 (1968), 492-517; CW 33, DOI 10.2307/1970722. MR0236190

[6] J. Tate, Duality theorems in Galois cohomology over number fields, Proc. Internat. Congr. Mathematicians (Stockholm, 1962), Inst. Mittag-Leffler, Djursholm, 1963, pp. 288-295; CW 18. MR0175892

[7] J. T. Tate, Algebraic cycles and poles of zeta functions, Arithmetical Algebraic Geometry (Proc. Conf. Purdue Univ., 1963), Harper \& Row, New York, 1965, pp. 93-110; CW 21. MR.0225778

[8] J. Tate, The cohomology groups of tori in finite Galois extensions of number fields, Nagoya Math. J. 27 (1966), 709-719; CW 25. MR.0207680

[9] J. Tate, Rigid analytic spaces, Invent. Math. 12 (1971), 257-289; CW 36, DOI 10.1007/BF01403307. MR0306196

[10] J. Tate, Symbols in arithmetic, Actes du Congrès International des Mathématiciens (Nice, 1970), Gauthier-Villars, Paris, 1971, pp. 201-211; CW 35. MR0422212

[11] J. Tate, Galois cohomology, Arithmetic algebraic geometry (Park City, UT, 1999), IAS/Park City Math. Ser., vol. 9, Amer. Math. Soc., Providence, RI, 2001, pp. 465-479; CW 74. MR.1857470

Department of Mathematics, University of Michigan, Ann Arbor, Michigan 